\title{
Contradiction between Conservation Laws and Orthodox Quantum Mechanics
}

\author{
M. E. Burgos
}

Departamento de Física, Facultad de Ciencias, Universidad de Los Andes, Mérida, Venezuela

Email: mburgos25@gmail.com

Received February $28^{\text {th }}, 2010$; revised April $7^{\text {th }}, 2010$; accepted May $7^{\text {th }}, 2010$.

\begin{abstract}
In this paper, it showed that the orthodox version of quantum mechanics contradicts the idea that conservation laws are valid in individual processes of measurement.
\end{abstract}

Keywords: Conservation Laws, Orthodox Quantum Mechanics, Measuring Problem

\section{Introduction}

The Schrödinger evolution of a system leads, in some circumstances, to coherent superpositions of macroscopically distinct states. This is dramatically illustrated in Schrödinger's cat paradox, and constitutes the great puzzle of quantum measurements.

To explain this fact, several hypotheses have been proposed. The best known is the projection postulate, an ingredient of the so-called orthodox interpretation of quantum mechanics (due to von Neumann), which is at present almost the only version taught. The projection postulate establishes that when a measurement is performed, the system's state jumps to an eigenstate of the operator representing the dynamical variable measured, and the pointer of the measuring device is led to a definite position; i.e., it breaks down the coherent superposition of macroscopically distinct states.

This postulate has been criticized on several grounds:

- it introduces a subjective element into the theory $[1,2]$,

- it conflicts with the Schrödinger equation $[2,3]$, and

- it implies a kind of action-at-a-distance $[2,4]$.

The traditionally opposed approach faces the conceptual difficulties of the measurement problem by assuming that the state function $\left|\Phi_{\mathrm{S}}\right\rangle$ is no more than a tool to calculate probabilities. Differing from the orthodox version, in this view $\left|\Phi_{\mathrm{S}}\right\rangle$ is not an attribute of an individual system $\mathrm{S}$ but of an ensemble; hence a process state reduction is not required [1]. Nevertheless, many physicists think that $\left|\Phi_{\mathrm{S}}\right\rangle$ refers to an individual system, so the ensemble interpretation of $\left|\Phi_{\mathrm{S}}\right\rangle$ that allows rejection of the projection postulate is, paradoxically, the main reason that this approach is frequently discarded.
In order to find a solution to the measuring problem keeping as valid the individual interpretation of $\left|\Phi_{\mathrm{S}}\right\rangle$, other theories close to, but different from, quantum mechanics have been proposed. In these theories, the Schrödinger equation is modified in a way that leads to spontaneous collapses. This is the case of those developed by Ghiradi, Rimini and Weber [5], Diosi [6], and Joos and Zeh [7]. Ballentine [8] has demonstrated that these theories violate energy conservation and are incompatible with the existence of stationary states.

Several authors [9-13] have studied the role of conservation laws in quantum measurements. It has been shown that the presence of an additive conserved quantity imposes restrictions on the measurement of dynamical variables incompatible with this quantity. The main object of the present paper is to point out an even deeper conflict between conservation laws and the orthodox version of quantum mechanics: if the individual interpretation of $\left|\Phi_{\mathrm{S}}\right\rangle$ and the projection postulate are taken as valid, then conservation laws cannot be satisfied in measurement processes, except in cases where the initial state of S is an eigenstate of the operator representing the quantity to be measured.

\section{Conservation Laws in Processes Involving an Individual System}

In the framework of classical physics, in principle, the application and test of conservation laws does not present any difficulty. This is mainly due to the fact that physical quantities have definite values. So if the numerical value $a$ of a physical quantity $A$ does not change during the whole process, we can assert that $A$ is conserved in this process. 
The same is valid in the statistical version of quantum mechanics [1].

On the contrary, in the framework of orthodox quantum mechanics, in general, dynamical variables (or physical quantities) are not sharp. "A popular working rule of pragmatic quantum mechanics says that an observable has no value before a measurement." [14] But nobody has stated, to our knowledge, in which way a conservation law should be applied or tested in those cases where the dynamical variable $A$ does not have a definite value. So if the orthodox interpretation is adopted, it is not evident in which way it could be decided whether $A$ is conserved or not. This means that in this version of quantum mechanics, a priori it does not make any sense to say that a dynamical variable which is not sharp, is conserved, even if the process follows the Schrödinger equation.

This radical conclusion can be avoided in the following way: If the operator $A_{S}$ represents the dynamical variable $A_{S}$ referred to the individual system $\mathrm{S}$ and $\mathrm{H}_{\mathrm{S}}$ is its Hamiltonian, in processes that are ruled by the Schrödinger equation, the conditions

$$
\partial \mathrm{A}_{\mathrm{S}} / \partial \mathrm{t}=0
$$

and

$$
\left[\mathrm{A}_{\mathrm{S}}, \mathrm{H}_{\mathrm{S}}\right]=0
$$

ensure that

$$
\left\langle\mathrm{A}_{\mathrm{S}}\right\rangle=\left\langle\Phi_{\mathrm{S}}\left|\mathrm{A}_{\mathrm{S}}\right| \Phi_{\mathrm{S}}\right\rangle
$$

remains a constant in time for every state $\left|\Phi_{\mathrm{S}}\right\rangle$ of $\mathrm{S}$.

Messiah postulates that the mean value of the dynamical variable $A_{\mathrm{S}}$ is $\left\langle\mathrm{A}_{\mathrm{S}}\right\rangle$ [15]. Taking into account this postulate we shall claim that if $A_{\mathrm{S}}$ is conserved, then $\left\langle\mathrm{A}_{\mathrm{S}}\right\rangle$ cannot change with time for every state $\left|\Phi_{\mathrm{S}}\right\rangle$ of $\mathrm{S}$. Hence, in those processes that are governed by the Schrödinger equation, the statement " $A_{S}$ is conserved" can be given a meaning, whether $A_{S}$ is sharp or not.

On one hand, let us stress that a necessary condition for the dynamical variable $A_{S}$ to be conserved is that $\left\langle\mathrm{A}_{\mathrm{S}}\right\rangle$ be a constant (observe, nevertheless, that this does not imply that $A_{S}$ takes on the value $\left.\left\langle\mathrm{A}_{\mathrm{S}}\right\rangle\right)$. On the other hand, it should be emphasised that in the framework of the version of quantum mechanics that we are analysing, both $\left|\Phi_{S}\right\rangle$ and $A_{\mathrm{S}}$ refer to the individual system $\mathrm{S}$. As a consequence, the quantity $\left\langle\mathrm{A}_{\mathrm{S}}\right\rangle$ given by (3) cannot concern something different from this individual system. This quantity is called expectation value by some authors and mean value by other authors. Since some people do not conceive that a mean value can be related to an individual system, let us quote some orthodox authors saying that $\left\langle\mathrm{A}_{\mathrm{S}}\right\rangle$ refers to an individual system.

a) According to von Neumann, the main architect of orthodox quantum mechanics, "everything which can be said about the state of the system must be derived from its wave function $\Phi$. What pronouncements can now be made regarding a system which is in the state $\Phi ? .$. For the expectation value of $\mathrm{R}$ in the state $\Phi$, we have $(\mathrm{R} \Phi, \Phi)[=$ $\langle\Phi|\mathrm{R}| \Phi\rangle]$ (emphases added)." [16]

b) In Messiah's words, "the mean value of the dynamical variable $A$ when the system is in the dynamical state defined by the [normalised] function $\Psi$ is $\langle\mathrm{A}\rangle=\langle\Psi$, A $\Psi>$ (emphasis added)." [15]

c) Merzbacher calls expectation value the quantity $\langle\mathrm{X}\rangle=$ $\langle\Phi|X| \Phi\rangle$. This author points out that "in quantum mechanics the term 'expectation value' is preferred when it is desirable to emphasise... the fact that the behaviour of $a$ single particle is involved rather than that of an ensemble of particles (emphasis added)." [17]

d) Cohen-Tannoudji et al. use expressions like "the mean value $\langle\mathrm{X}\rangle(\mathrm{t})$ of the position of the particle at time t..." and "the mean value of the energy of the particle in the state $|\Psi(\mathrm{t})\rangle_{\ldots .}$ (emphases added)." [18]

The precedent list of authors considering that the expectation (or mean) value refers to an individual system is not exhaustive. But it suffices, we think, to show that in general authors adopting the individual interpretation of $\left|\Phi_{S}\right\rangle$ assert that $\left\langle\mathrm{A}_{\mathrm{S}}\right\rangle=\left\langle\Phi_{\mathrm{S}}\left|\mathrm{A}_{\mathrm{S}}\right| \Phi_{\mathrm{S}}\right\rangle$ refers also to the individual system $\mathrm{S}$. In the following we are going to use the term mean value for individual systems, and the term $a v-$ erage when some set or ensemble is involved.

\section{Conservation Laws in Processes of Measurement (Case of a Discrete Spectrum)}

Now we shall address the problem of the validity of conservation laws when a measurement of $A_{\mathrm{S}}$ is performed. In this section we shall deal with the discrete case and, in the next one, with the continuous case. Let $\mathrm{a}_{\mathrm{k}}(\mathrm{k}=1,2, \ldots)$ be an eigenvalue of $A_{S}, g$ its degree of degeneracy and $\left|\mathrm{a}_{\mathrm{k}}^{\mathrm{i}}\right\rangle \quad(\mathrm{i}=1.2, \ldots \mathrm{g})$ an eigenvector corresponding to the eigenvalue $a_{k}$. We shall assume that $\left|m_{0}\right\rangle$ represents the initial state of a measuring device $\mathrm{M}$ of $A_{\mathrm{S}}$, and $\left|\psi_{\mathrm{k}}^{\mathrm{i}}\right\rangle$ the orthonormal states of $\mathrm{S}+\mathrm{M}$ when the measurement process is over. In the ideal measurement scheme, the transition

$$
\left|\mathrm{a}_{\mathrm{k}}^{\mathrm{i}}\right\rangle\left|\mathrm{m}_{0}\right\rangle \rightarrow\left|\psi_{\mathrm{k}}^{\mathrm{i}}\right\rangle
$$

has a probability of one. This scheme is supposed to be valid in cases where the measured dynamical variable is compatible with every conserved quantity referred to $\mathrm{S}+\mathrm{M}$ [9-13].

Let $\mathrm{A}$ be the operator representing a dynamical variable $A$ referred to $\mathrm{S}+\mathrm{M}$, and $\mathrm{H}$ be its Hamiltonian. We can then write

$$
\mathrm{H}=\mathrm{H}_{\mathrm{S}}+\mathrm{H}_{\mathrm{M}}+\mathrm{H}_{\mathrm{int}}
$$

where $\mathrm{H}_{M}$ refers to $\mathrm{M}$, and $\mathrm{H}_{\text {int }}$ is due to the interaction between $\mathrm{S}$ and $\mathrm{M}$. We assume that the conditions 


$$
\partial \mathrm{A} / \partial \mathrm{t}=0
$$

and

$$
[\mathrm{A}, \mathrm{H}]=0
$$

are fulfilled.

To ensure that measurements of $A_{\mathrm{S}}$ can be performed according to the ideal scheme, we suppose that $A_{S}$ commutes with every operator representing another conserved quantity referred to $\mathrm{S}+\mathrm{M}$; and, since the transition (4) has a probability of one, it can be assumed that it is a result of the Schrödinger evolution.

If at $t_{0}$ (when the interaction between $\mathrm{S}$ and $\mathrm{M}$ starts) it is possible to write

$$
\mathrm{A}=\mathrm{A}_{\mathrm{S}}+\mathrm{A}_{\mathrm{M}}
$$

(where $A_{M}$ refers to $M$ ), we have

$$
\langle\mathrm{A}\rangle_{\mathrm{k}}^{\mathrm{i}}\left(\mathrm{t}_{0}\right)=\left\langle\mathrm{a}_{\mathrm{k}}^{\mathrm{i}}\left|\mathrm{A}_{\mathrm{S}}\right| \mathrm{a}_{\mathrm{k}}^{\mathrm{i}}\right\rangle+\left\langle\mathrm{m}_{0}\left|\mathrm{~A}_{\mathrm{M}}\right| \mathrm{m}_{0}\right\rangle=\mathrm{a}_{\mathrm{k}}+\left\langle\mathrm{m}_{0}\left|\mathrm{~A}_{\mathrm{M}}\right| \mathrm{m}_{0}\right\rangle
$$

And, since at $t_{\mathrm{f}}$ (when the interaction between $\mathrm{S}$ and $\mathrm{M}$ is over)

$$
\langle\mathrm{A}\rangle_{\mathrm{k}}^{\mathrm{i}}\left(\mathrm{t}_{\mathrm{f}}\right)=\left\langle\psi_{\mathrm{k}}^{\mathrm{i}}|\mathrm{A}| \psi_{\mathrm{k}}^{\mathrm{i}}\right\rangle
$$

the validity of (6) and (7) implies that $\langle A\rangle_{k}^{i}\left(t_{f}\right)=$ $\langle\mathrm{A}\rangle_{\mathrm{k}}^{\mathrm{i}}\left(\mathrm{t}_{0}\right)$, and hence

$$
\left\langle\psi_{\mathrm{k}}^{\mathrm{i}}|\mathrm{A}| \psi_{\mathrm{k}}^{\mathrm{i}}\right\rangle=\mathrm{a}_{\mathrm{k}}+\left\langle\mathrm{m}_{0}\left|\mathrm{~A}_{\mathrm{M}}\right| \mathrm{m}_{0}\right\rangle
$$

for every i. As $\left\langle\psi_{k}^{i}|\mathrm{~A}| \psi_{\mathrm{k}}^{\mathrm{i}}\right\rangle$ does not depend on i, it can be written

$$
\langle A\rangle_{k}\left(t_{f}\right)=\langle A\rangle_{k}^{i}\left(t_{f}\right)=a_{k}+\left\langle m_{0}\left|A_{M}\right| m_{0}\right\rangle
$$

This relation must necessarily be fulfilled in the ideal measurement scheme. As a consequence, it can be said that in those cases where the initial state of $\mathrm{S}$ is an eigenstate of the operator $A_{S}$ representing the dynamical variable $A_{\mathrm{S}}$ to be measured, the corresponding conservation law is valid. This result can also be seen as a natural consequence of the hypothesis that the process described by (4) is governed by the Schrödinger equation.

Now, if the initial state of $\mathrm{S}$ is

$$
\left|\Phi_{\mathrm{s}}\left(\mathrm{t}_{0}\right)\right\rangle=\sum_{\mathrm{r}, \mathrm{i}} \mathrm{c}_{\mathrm{r}}^{\mathrm{i}}\left|\mathrm{a}_{\mathrm{r}}^{\mathrm{i}}\right\rangle
$$

(where at least two coefficients $c_{r}{ }^{i}$ and $c_{r^{\prime}}{ }^{\prime}$ with $r \neq r^{\prime}$ are non-null) and the Schrödinger equation rules the measurement process, then the Hamiltonian $\mathrm{H}$, referred to $\mathrm{S}+\mathrm{M}$, induces the evolution

$$
\left.\sum_{r, i} c_{r}^{i}\left|a_{r}^{i}\right\rangle\left|m_{0}\right\rangle \rightarrow \sum_{r, i} c_{r}^{i} \psi_{r}^{i}\right\rangle
$$

Making

$$
\langle\mathrm{A}\rangle\left(\mathrm{t}_{0}\right)=\left(\left\langle\Phi_{\mathrm{S}}\left(\mathrm{t}_{0}\right)\left|\left\langle\mathrm{m}_{0}\right|\right) \mathrm{A}\left(\left|\Phi_{\mathrm{S}}\left(\mathrm{t}_{0}\right)\right\rangle\left|\mathrm{m}_{0}\right\rangle\right)\right.\right.
$$

and

$$
\langle\mathrm{A}\rangle\left(\mathrm{t}_{\mathrm{f}}\right)=\left(\sum_{\mathrm{r}, \mathrm{i}} \mathrm{c}_{\mathrm{r}}^{\mathrm{i}^{*}}\left\langle\psi_{\mathrm{r}}^{\mathrm{i}}\right|\right) \mathrm{A}\left(\sum_{\mathrm{r}^{\prime},}, \mathrm{e}_{\mathrm{r}}, \mathrm{i}_{\mathrm{r}}^{\mathrm{i}}\left|\psi_{\mathrm{r}^{\prime}}^{\mathrm{i}^{\mathrm{i}}}\right\rangle\right)
$$

the validity of (6) and (7) allow us to ensure that $\langle\mathrm{A}\rangle\left(\mathrm{t}_{0}\right)=$ $\langle A\rangle\left(t_{\mathrm{f}}\right)$. Nevertheless, the linear superposition on the r.h. of (14), mentioned in Section 1, constitutes the great puzzle of quantum measurements.

On the contrary, the projection postulate states that in measurement processes coherent superpositions break down. According to this postulate, the evolution of $\mathrm{S}+\mathrm{M}$ is not given by (14) and the transition

$$
\sum_{\mathrm{r}, \mathrm{i}} \mathrm{c}_{\mathrm{r}}^{\mathrm{i}}\left|\mathrm{a}_{\mathrm{r}}^{\mathrm{i}}\right\rangle\left|\mathrm{m}_{0}\right\rangle \rightarrow \sum_{\mathrm{i}} \mathrm{c}_{\mathrm{k}}^{\mathrm{i}}\left|\psi_{\mathrm{k}}^{\mathrm{i}}\right\rangle
$$

has probability $\sum_{\mathrm{i}}\left|\mathrm{c}_{\mathrm{k}}^{\mathrm{i}}\right|^{2}$ to happen. In this last case,

$$
\langle\mathrm{A}\rangle\left(\mathrm{t}_{0}\right)=\sum_{\mathrm{i}}\left|\mathrm{c}_{\mathrm{k}}^{\mathrm{i}}\right|^{2} \mathrm{a}_{\mathrm{r}}+\left\langle\mathrm{m}_{0}\left|\mathrm{~A}_{\mathrm{M}}\right| \mathrm{m}_{0}\right\rangle
$$

and, as stated in (12),

$$
\langle\mathrm{A}\rangle_{\mathrm{k}}\left(\mathrm{t}_{\mathrm{f}}\right)=\mathrm{a}_{\mathrm{k}}+\left\langle\mathrm{m}_{0}\left|\mathrm{~A}_{\mathrm{M}}\right| \mathrm{m}_{0}\right\rangle
$$

As a consequence, it results

$$
\langle\mathrm{A}\rangle\left(\mathrm{t}_{0}\right) \neq\langle\mathrm{A}\rangle_{\mathrm{k}}\left(\mathrm{t}_{\mathrm{f}}\right)
$$

for every $k$, even though conditions (6) and (7) are fulfilled.

It is worth noticing that inequalities (20) are obtained under the assumptions that the individual interpretation of the state vector and the projection postulate are valid. In this case the condition that $\langle\mathrm{A}\rangle$ be a constant, a necessary condition for $A$ to be conserved, is not satisfied. We are thus forced to conclude that if the initial state of $\mathrm{S}$ is not an eigenvector of $A_{S}$, the dynamical variable $A$ is not conserved in processes of measurement of $A_{\mathrm{s}}$. In other articles we have given examples of processes of measurement of the type analyzed in this section [19-22].

A similar conclusion resulting from a different analysis has been obtained by Pearle [23]. He says that "it should first be noted that quantum theory itself, with the reduction postulate indiscriminately applied, does not necessarily satisfy the conservation laws..." In his view, "this is a serious problem for quantum theory with a reduction postulate."

We have said that $\langle\mathrm{A}\rangle\left(\mathrm{t}_{0}\right)=\sum_{\mathrm{r}, \mathrm{i}}\left|\mathrm{c}_{\mathrm{r}}^{\mathrm{i}}\right|^{2} \mathrm{a}_{\mathrm{r}}+\left\langle\mathrm{m}_{0}\left|\mathrm{~A}_{\mathrm{M}}\right| \mathrm{m}_{0}\right\rangle$. Now we are going to calculate the average of $\langle\mathrm{A}\rangle_{\mathrm{k}}\left(\mathrm{t}_{\mathrm{f}}\right)$ when the process of measurement of $A_{S}$ is repeated $\mathrm{N}$ times. Let $f_{k}$ be the frequency corresponding to the possible results $a_{k}(k=1,2, \ldots)$ and to the mean value $\langle\mathrm{A}\rangle_{\mathrm{k}}\left(\mathrm{t}_{\mathrm{f}}\right)$. If the process is repeated $\mathrm{N}$ times, the resulting average is

$$
\overline{\mathrm{A}}=\sum_{\mathrm{k}} \mathrm{f}_{\mathrm{k}}\langle\mathrm{A}\rangle_{\mathrm{k}}\left(\mathrm{t}_{\mathrm{f}}\right)
$$

and, taking into account (19),

$$
\overline{\mathrm{A}}=\sum_{\mathrm{k}} \mathrm{f}_{\mathrm{k}} \mathrm{a}_{\mathrm{k}}+\left\langle\mathrm{m}_{0}\left|\mathrm{~A}_{\mathrm{M}}\right| \mathrm{m}_{0}\right\rangle .
$$

Now, if $\mathrm{N}$ is big enough, we can assert that $\mathrm{f}_{\mathrm{k}} \approx \sum_{\mathrm{i}}\left|\mathrm{c}_{\mathrm{k}}^{\mathrm{i}}\right|^{2}$. As a consequence, we obtain 


$$
\begin{gathered}
\overline{\mathrm{A}} \approx \sum_{\mathrm{k}, \mathrm{i}}\left|\mathrm{c}_{\mathrm{k}}^{\mathrm{i}}\right|^{2} \mathrm{a}_{\mathrm{k}}+\left\langle\mathrm{m}_{0}\left|\mathrm{~A}_{\mathrm{M}}\right| \mathrm{m}_{0}\right\rangle \\
\langle\mathrm{A}\rangle\left(\mathrm{t}_{0}\right)=\sum_{\mathrm{k}, \mathrm{i}}\left|\mathrm{c}_{\mathrm{k}}^{\mathrm{i}}\right|^{2} \mathrm{a}_{\mathrm{k}}+\left\langle\mathrm{m}_{0}\left|\mathrm{~A}_{\mathrm{M}}\right| \mathrm{m}_{0}\right\rangle
\end{gathered}
$$

and

$$
\overline{\mathrm{A}} \approx\langle\mathrm{A}\rangle\left(\mathrm{t}_{0}\right)
$$

So we can say that conservation laws still have a statistical sense.

\section{Conservation Laws in Processes of Measurement (Case of a Continuous Spectrum)}

Let $\alpha$ be an eigenvalue included in the continuous part of the spectrum of $\mathrm{A}_{S}$; we shall assume that $\alpha$ can take any value in the interval $(0, \infty)$. If the ket $|\alpha\rangle$ is

$$
|\alpha\rangle=\int_{\alpha-\delta / 2}^{\alpha+\delta / 2} \operatorname{dac}(\mathrm{a})|\mathrm{a}\rangle
$$

where $\delta$ is a small interval in the $\alpha$ semi-axis (if $\Delta \alpha$ is the error in the measurement of $\alpha$, the condition $\delta<\Delta \alpha$ should be fulfilled), we shall say that $|\alpha\rangle$ is a "quasi-eigenstate" of $A_{S}$ corresponding to the eigenvalue $\alpha$. We shall call $\left|\mathrm{m}_{0}\right\rangle$ the ket that represents the initial state of a measuring device $\mathrm{M}$ of $A_{\mathrm{S}}$, and $|\psi(\alpha)\rangle$ the orthonormal states of $\mathrm{S}+\mathrm{M}$ when the process of measurement is over. If the initial state of $S$ is $|\alpha\rangle$, according to the ideal measurement scheme, the transition

$$
|\alpha\rangle\left|\mathrm{m}_{0}\right\rangle \rightarrow|\psi(\alpha)\rangle
$$

has a probability of one.

Let A be the operator representing a dynamical variable $A$ referred to $\mathrm{S}+\mathrm{M}$, and $\mathrm{H}$ be its Hamiltonian. We can then write

$$
\mathrm{H}=\mathrm{H}_{\mathrm{S}}+\mathrm{H}_{\mathrm{M}}+\mathrm{H}_{\text {int }}
$$

where $\mathrm{H}_{M}$ refers to $M$, and $\mathrm{H}_{\text {int }}$ is due to the interaction between $\mathrm{S}$ and $\mathrm{M}$. As previously, we assume that the conditions (6) and (7) are fulfilled.

If at $\mathrm{t}_{0}$ (when the interaction between $\mathrm{S}$ and $\mathrm{M}$ starts) it is possible to write

$$
A=A_{S}+A_{M}
$$

(where $A_{M}$ refers to $M$ ), we have

$$
\langle\mathrm{A}\rangle_{\alpha}\left(\mathrm{t}_{0}\right)=\left\langle\alpha\left|\mathrm{A}_{\mathrm{S}}\right| \alpha\right\rangle+\left\langle\mathrm{m}_{0}\left|\mathrm{~A}_{\mathrm{M}}\right| \mathrm{m}_{0}\right\rangle
$$

And since at $t_{\mathrm{f}}$ (when the interaction between $\mathrm{S}$ and $\mathrm{M}$ is over)

$$
\langle\mathrm{A}\rangle_{\alpha}\left(\mathrm{t}_{\mathrm{f}}\right)=\langle\psi(\alpha)|\mathrm{A}| \psi(\alpha)\rangle
$$

the validity of (6) and (7) implies that $\langle\mathrm{A}\rangle_{\alpha}\left(\mathrm{t}_{\mathrm{f}}\right)=\langle\mathrm{A}\rangle_{\alpha}\left(\mathrm{t}_{0}\right)$, and hence

$$
\langle\mathrm{A}\rangle_{\alpha}\left(\mathrm{t}_{\mathrm{f}}\right)=\left\langle\alpha\left|\mathrm{A}_{\mathrm{S}}\right| \alpha\right\rangle+\left\langle\mathrm{m}_{0}\left|\mathrm{~A}_{\mathrm{M}}\right| \mathrm{m}_{0}\right\rangle
$$

This relation must necessarily be fulfilled in the ideal measurement scheme. As a consequence, it can be said that in cases where the initial state of $\mathrm{S}$ is a "quasi- eigenstate" of $A_{S}$, the corresponding conservation law is valid.

Now, if the initial state of $\mathrm{S}$ is

$$
\left|\Phi_{\mathrm{s}}\left(\mathrm{t}_{0}\right)\right\rangle=\int_{0}^{\infty} \mathrm{dac}(\mathrm{a})|\mathrm{a}\rangle
$$

where $c(a) \neq 0$ outside the interval $(\alpha-\delta / 2, \alpha+\delta / 2)$, and the Schrödinger equation rules the measurement process, then the Hamiltonian $\mathrm{H}$ induces the evolution

$$
\begin{aligned}
& \int_{0}^{\infty} \mathrm{dac}(\mathrm{a})|\mathrm{a}\rangle\left|\mathrm{m}_{0}\right\rangle \rightarrow \int_{0}^{\infty} \mathrm{dac}(\mathrm{a})|\psi(\mathrm{a})\rangle \\
& \quad=\int_{0}^{\alpha-\delta / 2} \operatorname{dac}(\mathrm{a})|\psi(\mathrm{a})\rangle+\int_{\alpha-\delta / 2}^{\alpha+\delta / 2} \mathrm{dac}(\mathrm{a})|\psi(\mathrm{a})\rangle \\
& \quad+\int_{\alpha+\delta / 2}^{\infty} \operatorname{dac} \text { ( a ) }|\psi(\mathrm{a})\rangle
\end{aligned}
$$

As a consequence, making

$$
\langle\mathrm{A}\rangle\left(\mathrm{t}_{0}\right)=\left(\left\langle\Phi_{\mathrm{S}}\left(\mathrm{t}_{0}\right)\left|\left\langle\mathrm{m}_{0}\right|\right) \mathrm{A}\left(\left|\Phi_{\mathrm{S}}\left(\mathrm{t}_{0}\right)\right\rangle\left|\mathrm{m}_{0}\right\rangle\right)\right.\right.
$$

and

$$
\left.\langle\mathrm{A}\rangle\left(\mathrm{t}_{\mathrm{f}}\right)=\left[\int_{0}^{\infty} \mathrm{dac}^{*}(\mathrm{a})\langle\psi(\mathrm{a})|\right]+\int_{0}^{\infty} \mathrm{da} \mathrm{a}^{\prime} \mathrm{c}\left(\mathrm{a}^{\prime}\right)\left|\psi\left(\mathrm{a}^{\prime}\right)\right\rangle\right]
$$

the validity of (6) and (7) allow us to ensure that $\langle\mathrm{A}\rangle\left(\mathrm{t}_{0}\right)=$ $\langle\mathrm{A}\rangle\left(\mathrm{t}_{\mathrm{f}}\right)$. But, as it happened in the case of the discrete spectrum, we obtain a linear superposition in the r.h. of (34), previously mentioned, and that constitutes the great puzzle of quantum measurements.

On the contrary, the projection postulate states that in measurement processes coherent superpositions break down. According to this postulate, the evolution of $\mathrm{S}+\mathrm{M}$ is not given by (34), and the transition

$$
\int_{0}^{\infty} \mathrm{dac}(\mathrm{a})|\mathrm{a}\rangle\left|\mathrm{m}_{0}\right\rangle \rightarrow \int_{\alpha-\delta / 2}^{\alpha+\delta / 2} \operatorname{dac}(\mathrm{a})|\psi(\mathrm{a})\rangle \int_{0}^{\infty}
$$

has a probability close to $|\mathrm{c}(\alpha)|^{2} \delta$ to happen. So, since

$$
\langle\mathrm{A}\rangle\left(\mathrm{t}_{0}\right)=\int_{0}^{\infty} \mathrm{da}|\mathrm{c}(\mathrm{a})|^{2} \mathrm{a}+\left\langle\mathrm{m}_{0}\left|\mathrm{~A}_{\mathrm{M}}\right| \mathrm{m}_{0}\right\rangle
$$

and

it results

$$
\begin{aligned}
\langle\mathrm{A}\rangle_{\alpha}\left(\mathrm{t}_{\mathrm{f}}\right) & =\int_{\alpha-\delta / 2}^{\alpha+\delta / 2} \mathrm{da}|\mathrm{c}(\mathrm{a})|^{2} \mathrm{a}+\left\langle\mathrm{m}_{0}\left|\mathrm{~A}_{\mathrm{M}}\right| \mathrm{m}_{0}\right\rangle \\
& =\alpha+\left\langle\mathrm{m}_{0}\left|\mathrm{~A}_{\mathrm{M}}\right| \mathrm{m}_{0}\right\rangle
\end{aligned}
$$

$$
\langle\mathrm{A}\rangle\left(\mathrm{t}_{0}\right) \neq\langle\mathrm{A}\rangle_{\alpha}\left(\mathrm{t}_{\mathrm{f}}\right)
$$

for every $\alpha$, even though conditions (6) and (7) are fulfilled.

It is worth noticing that inequalities (20) and (40) are obtained under the assumptions that the individual interpretation of the state vector and the projection postulate are valid. In this case the condition that $\langle\mathrm{A}\rangle$ be a constant, a necessary condition for $A$ to be conserved, is not satisfied. We are thus forced to conclude that if the initial state of $S$ is not an eigenvector of $A_{S}$ (in the discrete case) or a "quasi-eigenvector" of $A_{S}$ (in the continuous case), the dynamical variable $A$ is not conserved in measurement processes of $A_{\mathrm{S}}$. The proof that also in this last case conservation laws still have a 
statistical sense is straightforward.

\section{Concluding Remarks}

We have seen that during Schrödinger evolutions, the validity of (6) and (7) ensures that the expectation value $\langle\mathrm{A}\rangle$ referred to the individual system $\mathrm{S}$ and its measurement device $\mathrm{M}$ remains constant in time. But if the rule governing the process is replaced with a law different from Schrödinger equation, the validity of conservation laws cannot be guaranteed a priori [19-24]. Ballentine points out that some theories that modify the Schrödinger equation in order to include spontaneous state reductions lead to the non-conservation of the energy [8]. Our study shows that projections induced by measurements, as they are considered in the framework of orthodox quantum mechanics, conflict with the conservation laws.

However, the results of these two analyses have a difference worth noticing. In the theories Ballentine refers to, energy is continuously gained, although its magnitude is too small to be detected [8]. In collapses occurring in the framework of orthodox quantum mechanics, the change $\langle A\rangle\left(t_{f}\right)-\langle A\rangle\left(t_{0}\right)$ is not necessarily small but, when the process of measurement of $A_{\mathrm{S}}$ is repeated many times, the average of $\langle A\rangle\left(t_{f}\right)$ is close to $\langle A\rangle\left(t_{0}\right)$. This is why we claim that in measurement processes, conservation laws still have a statistical sense.

In an approach to quantum mechanics previously formulated we have included, as an essential ingredient, a postulate that ensures the statistical sense of conservation laws in every process involving projections $[25,26]$. In this approach no reference to the subject or to measurement devices is made. We there assume that in nature two kinds of spontaneous processes occur: those ruled by the Schrödinger equation, which is a deterministic equation, and those ruled by the rules of probability, where projections happen.

Let us conclude by pointing out that, in our view, there is nothing sacred about conservation laws. Like every other scientific law, they could be false. The same is true of the orthodox interpretation of quantum mechanics. The intent of our contribution is to show that there is a contradiction between these two ideas, both of which are adopted, perhaps, by the majority of physicists.

\section{Acknowledgements}

We thank Professor G. Díaz de Delgado for her help with stylistic matters. This work was supported by the CDCHT-ULA.

\section{REFERENCES}

[1] L. E. Ballentine, "The statistical interpretation of quantum mechanics," Reviews of Modern Physics, Vol. 42, 1970, p. 358.

[2] M. Bunge, “Treatise on Basic Philosophy," Vol. 7, Reidel, Dordrecht, 1985, pp. 191-205.
[3] P. Busch and A. Shimony, "Unsolubility of the Quantum Measurement Problem for Unsharp Unobservables," Physical Review D, Vol. 9, 1974, p. 2321.

[4] M. Jammer, "The Philosophy of Quantum Mechanics," John Wiley \& Sons, New York, 1974.

[5] G. C. Ghirardi, A. Rimini and T. Weber, "The Puzzling Entanglement of Schrödinger Wave Function," Foundations of Physis C, Vol. 18, No. 1, 1987, pp. 1-27.

[6] L. Diosi, "Models for Universal Reduction of Macroscopic Quantum Fluctuations," Physical Review A, Vol. 40, No. 3, 1989, pp. 1165-1174.

[7] E. Joos and H. D. Zeh, "The Emergence of Classical Properties through Interaction with the Enviroment," Zeitschrift für Physik B, Vol. 59, 1985, p. 223.

[8] L. E. Ballentine, "Failure of Some Theories of State Reduction," Physical Review A, Vol. 43, No. 1, 1991, pp. 9-12.

[9] E. P. Wigner, "Die Messung Quantenmechanischer Operatoren," Zeitschrift für Physik, Vol. 131, 1952, pp. 101-108.

[10] H. Araki and M. M. Yanase, "Measurement of Quantum Mechanical Operators," Physical Review, Vol. 120, No. 2, 1960, pp. 622-626.

[11] H. Stein and A. Shimony, "Limitation on Quantum Measurements," in: B. d'Espagnat, Ed., Foundations of Quantum Mechanics, Academic, New York, 1971.

[12] G. C. Ghirardi, F. Miglietta, A. Rimini and T. Weber, "Limitation on Quantum Measurements," Physical Review D, Vol. 24, No. 2, 1981, pp. 353-358.

[13] M. Ozawa, "Does a Conservation Law Limit Position Measurements?" Physical Review Letters, Vol. 67, No. 15, 1991, pp. 1956-1159.

[14] H. Primas, "Realism and Quantum Mechanics," Proceedings of 9th International Congress of Logic, Methodology and Philosophy of Science, Vol. 134, 1991, pp. 609-631.

[15] A. Messiah, "Quantum Mechanics," North-Holland Pub.lishing Company, Amsterdam, 1974.

[16] J. von Neumann, "Mathematical Foundations of Quantum Mechanics," Princeton University Press, Princeton, New Jersey, 1955.

[17] E. Merzbacher, "Quantum Mechanics," John Wiley and Sons, New York, 1977.

[18] C. Cohen-Tannoudji, B. Diu and F. Laloë, "Quantum Mechanics,” John Wiley and Sons, New York, 1977.

[19] M. E. Burgos, "Conservation Laws and Deterministic Evolutions," Physics Essays, Vol. 7, No. 1, 1994, pp. 69-71.

[20] M. E. Burgos, "Does Conservation of Energy Apply in Processes Ruled by Quantum Mechanical Laws?" Speculations in Science and Technology, Vol. 20, 1997, pp. 183-187.

[21] M. E. Burgos, F. G. Criscuolo and T. L. Etter, "Conservation Laws, Machines of The First Type and Superluminal Communication," Speculations in Science and Technology, Vol. 21, No. 4, 1999, pp. 227-233. 
[22] F. G. Criscuolo and M. E. Burgos, "Conservation Laws in Spontaneous and Mesurement-Like Individual Processes," Physics Essays, Vol. 13, No. 1, 2000, pp. 80-84.

[23] P. Pearle, "Suppose the State Vector is Real: The Description and Consequences of Dynamical Reduction," Annals of the New York Academy of Sciences, Vol. 480 1986, pp. 539-551.

[24] A. Afriat and F. Selleri, "The Foundations of Quantum Mechanics-Historical Analysis and Open Questions," in: C.
Garola and A. Rossi, Eds., Kluwer Academic Publishers, Dordrecht, 1995.

[25] M. E. Burgos, "Which Natural Processes Have the Special Status of Measurements?" Foundations of Physisc, Vol. 28, No. 8, 1998, pp. 1323-1346.

[26] M. E. Burgos, "Transitions to the Continuum: Three Different Approaches," Foundations of Physisc, Vol. 38, No. 10, 2008, pp. 883-907. 